\title{
Localized minimum-energy broadcasting in ad-hoc networks
}

\author{
Julien Cartigny and David Simplot \\ IRCICA/LIFL CNRS UPRESA 8022 \\ Université de Lille 1 \\ Cité Scientifique, Bât. M3 \\ 59655 Villeneuve d'Ascq Cedex, France \\ Email: \{Julien.Cartigny,David.Simplot\}@lifl.fr
}

\author{
Ivan Stojmenović \\ Computer Science, SITE \\ University of Ottawa \\ Ottawa, Ontario K1N 6N5, Canada \\ Email: ivan@site.uottawa.ca
}

\begin{abstract}
In the minimum energy broadcasting problem, each node can adjust its transmission power in order to minimize total energy consumption but still enable a message originated from a source node to reach all the other nodes in an ad-hoc wireless network. In all existing solutions each node requires global network information (including distances between any two neighboring nodes in the network) in order to decide its own transmission radius. In this paper, we describe a new localized protocol where each node requires only the knowledge of its distance to all neighboring nodes and distances between its neighboring nodes (or, alternatively, geographic position of itself and its neighboring nodes). In addition to using only local information, our protocol is shown experimentally to be comparable to the best known globalized BIP solution. Our solutions are based on the use of relative neighborhood graph which preserves connectivity and is defined in localized manner.
\end{abstract}

\section{INTRODUCTION}

In wireless ad-hoc networks, such as sensor networks, all nodes cooperate to handle network facilities. These networks are power constrained as nodes operate with restricted battery power. We consider nodes that have the capacity to modify the area of coverage with its transmission. Indeed, control of the emitted transmission power allows to reduce significantly the energy consumption and so to increase lifetime of the network. However, the adjustment of transmission signal strength generally implies topology alterations like loss of the connectivity. Hence, nodes have to manage their transmission area while maintaining the connectivity of the network.

In the broadcasting task, a message originated from a source node needs to be forwarded to all the other nodes in the network. In this paper, we focus on the development of protocols for energy-efficient broadcast communications. All existing solutions are globalized, meaning that each node needs global network information. Mobility of nodes, or changes in their activity status (from active to passive and vice versa) may cause global changes in any MST based structure. Therefore topology changes must be propagated throughout the network for any globalized solution. This may result in extreme and unacceptable communication overhead for ad-hoc networks. Hence, because of the limited resources of mobile nodes, it is ideal that each node can decide on its own behavior based only on the information from all nodes within a constant hop distance. Such distributed algorithms and protocols are called localized [1], [2], [3], [4], [5]. Of particular interest are protocols where nodes make decisions based solely on the knowledge of its 1-hop or 2-hops neighbors, and distances to them. In non-localized distributed, or globalized, algorithms nodes require knowledge of whole network topology to make decision.

Several different protocols have been proposed to manage energy consumption by adjusting transmitting powers. Among existing protocols, we can distinguish two families of protocols: topology control oriented protocols and broadcast oriented protocols.

The first family (topology control oriented protocols) assigns the transmission power for each node such that the network is connected independently of broadcast utilization. That means that all nodes can be a source of a broadcast and are able to reach all nodes of the network using pre-assigned transmission radii at each node. The optimization criterion is minimizing the total transmission power assigned according to an energy consumption model. This problem is known as min(total) assignment problem and was considered by Kiroustis et al. [6] which established that this problem is NP-hard for tree-dimensional space. Clementi et al. [7] showed that this complexity result still occurs for two-dimensional space. Approximate solutions [2], [8], [9] are based on minimum spanning trees or approximation of minimal spanning trees and are globalized.

The second family (broadcast oriented protocols) achieves the same objectives but considers the broadcast process from a given source node. For instance, Wieselthier et al. [9] proposed greedy heuristics which are based on Prim's and Dijkstra's algorithms. The more efficient heuristic, called BIP for broadcasting incremental power, constructs a tree starting from the source node and adds new nodes one at a time according to a cost evaluation. The constraints are not the same as for the first protocol family since in this second case the subgraph induced by the minimum-energy broadcast tree does not need to be strongly connected: the only condition is that the source can reach every node of the network. It has been proved in [10], [11] that the minimum-energy broadcast tree 
problem is NP-complete and [11] proposed an approximate globalized algorithm which gives solutions with bounded ratio against lower bound.

We can also distinguish several communication models: one-to-all model, one-to-one model and variable angular range model. In one-to-all model, mobile nodes use omnidirectional antennas and the communication zone of a node is a disk centered at this node. All above cited works (and all references except [12], [13], [14]) use this model. In one-to-one model, nodes are equipped with directional antennas with small angles that can provide more energy savings and interference reduction since the communication zone of a node is a small beam from this node to the targeted node [13]. With variable angular range model, the nodes can choose direction and width of the beam that allows to target several neighbor with one transmission. Hardware solutions using directional antennas (also called smart antennas) are more difficult to implement and we focus in this paper on one-to-all model. The broadcast energy problem for other models are addressed in [14], [12].

In this paper we are mainly interested in broadcast oriented protocols in one-to-all communication model in wireless adhoc networks. The main contribution of this paper is that we propose an algorithm that requires local information while all existing solutions are globalized, that is distributed where nodes require full knowledge of network to make decision. The information needed in our protocols are included in information needed by existing protocols like BIP. In our localized protocols, each node requires only the knowledge of its distance to all neighboring nodes and distances between its neighboring nodes. Distances can be measured by using signal strength, time delay or more sophisticated techniques like microwave distance [15]. If a positioning system (like GPS) is available, each node only needs position information from its neighbor nodes.

The paper is organized as follows. In next section, we present communication and energy models. In Section III, we give a literature review of minimum energy broadcast protocols. In Section IV, we describe how this problem can be solved with localized algorithms. Section V presents the results of our simulations where we demonstrate the efficiency and superiority of our algorithms. Finally, Section VI presents conclusion and future directions.

\section{Preliminaries}

\section{A. Communication Model}

We consider multi-hop wireless networks where all nodes cooperate in order to fulfill a given communication task. Such a network can be modeled as follows. A wireless network is represented by a graph $G=(V, E)$ where $V$ is the set of nodes and $E \subseteq V^{2}$ the edge set which gives the available communications: $(u, v)$ belongs to $E$ means that $u$ can send messages to $v$. In fact, elements of $E$ depend of node positions and communicating range of nodes. Let us assume that maximum range of communication, denoted by $R$, is the same for all vertices and that $d(u, v)$ is the distance between nodes $u$ and $v$.

For instance, the set $E$ can be defined as follows:

$$
E=\left\{(u, v) \in V^{2} \mid d(u, v) \leq R\right\} .
$$

So defined graph is known as the unit graph, with $R$ as its transmission radius.

In given graph $G=(V, E)$, we denote by $n=|V|$ the number of nodes in ad-hoc network. The neighbor set $N(u)$ of vertex $u$ is defined as $N(u)=\{v \mid(u, v) \in E\}$. The average degree of the network is the average number of neighbors of its nodes.

We will assume that each node can change the power of its transmissions for energy savings reasons (see next subsection). In this case, the range of a node $u \in V$ represents the maximal distance between $u$ and a node which can receive its transmission. The range of a node $u \in V$ is denoted by $r(u)$ (with $0 \leq r(u) \leq R$ ). The graph induced by the range assignment function $r$ is denoted by $G_{r}=\left(V, E_{r}\right)$ where the edge set $E_{r}$ is defined by:

$$
E_{r}=\left\{(u, v) \in V^{2} \mid d(u, v) \leq r(u)\right\} .
$$

It is straightforward to see that the graph $G_{r}$ with modified ranges is not always undirectional.

A (directed) graph is strongly connected if for any two vertices $u$ and $v$, a path connecting $u$ to $v$ exists. In the broadcasting task, a message needs to reach all nodes in the network by transmitting from the source and retransmitting by other network nodes with variable transmission radii. Hence, in case of broadcast, the strong connectivity is not needed, we only need connectivity from source node to all the other nodes in the network.

\section{B. Energy Model}

Commonly, the measurement of the energy consumption of network interfaces when transmitting a unit message depends on the range of the emitter $u$ :

$$
E(u)=r(u)^{\alpha},
$$

where $\alpha$ is a real constant greater than 2 and $r(u)$ is the range of the transmitting node. This model is used in [16], [2], [10], [17], [8], [18], [19], [9]. In reality, however, it has a constant to be added in order to take into account the overhead due to signal processing, minimum energy needed for successful reception and MAC control messages [20]. The general energy consumption formula is:

$$
E(u)=\left\{\begin{array}{lr}
r(u)^{\alpha}+c & \text { if } r(u) \neq 0, \\
0 & \text { otherwise. }
\end{array}\right.
$$

For instance, Rodoplu and Meng [21] consider the model with $E(u)=r(u)^{4}+10^{8}$. This last model, also used in [22], is more realistic as illustrated is Fig. 1: with parameters $\alpha=2$ and $c=0$, it is clear that the transmissions illustrated in subfigure (b) cost the same energy as the one in subfigure 
(a) by using Pythagoras theorem. By induction, all illustrated configurations are supposed to have the same energy consumption and can be arbitrary extended. For medium access, signal processing and reception power reason, it is not in accordance with real world.

Another example are nodes placed on a line segment. Assuming $c=0$ and $\alpha \geq 2$, it follows that energy savings are obtained when arbitrary number of nodes are placed between source $S$ and destination $D$, and these nodes are used to retransmit the message. This will certainly contradict basic signal processing requirement for minimal reception power, and cause significant amount of collisions in medium access layer if used by many simultaneous routing, multicasting and broadcasting tasks.

\section{Minimum energy broadcasting}

A transmission range assignment on the vertices in $V$ is a function $r$ from $V$ into an real interval $[0, R]$ where $R$ is the maximal range of nodes. In some wireless networks, the transmission range at each node has finite number of possible values meaning that $r$ is a function into a finite subset of $\mathbb{R}$. In accordance to reviewed literature, each node can adjust its own power level, i.e. that can adjust its transmission range. Each node has to reduce its transmission range while maintaining the connectivity of the graph. The measurement of total power consumption is given by the following formula:

$$
E=\sum_{u \in V} E(u) .
$$

\section{LITERATURE REVIEW}

We start with topology control protocols that aim to adjust transmission power while preserving strong connectivity of the network. In [6], the Kirousis et al. address the tree construction in wireless networks by using globalized protocols. The authors showed that this problem is NP-hard for three dimensional space and give an approximation algorithm for constructing a spanning tree that minimizes the total power consumption. Clementi et al. showed that the minimum energy range assignment problem is still NP-hard in two-dimensional case.

Wieselthier et al. define in [9] a topology control algorithm based on minimum-power spanning tree (MST in short). Let $V$ be a set of nodes and $G=(V, E)$ the induced graph with maximal range $R$. We assume that the graph $G$ is strongly connected. The weights of edges are given by the selected energy model (but in fact, the MST does not depend on particular choice of the metric because of monotonicity). The construction of the MST is possible if we can determine distances between nodes. For instance, Fig. 2 and 3 show a graph of 100 vertices and its MST. Notice that in the unit graph on Fig. 2 the average degree is 8 while in the MST on Fig. 3, the average degree is less than 2 .

It is well-known that the graph $M S T(G)=\left(V, E_{m s t}\right)$ of the MST is symmetric (undirected). It is easy to see that every node of $V$ can be a root of a spanning tree by using $M S T(G)$.

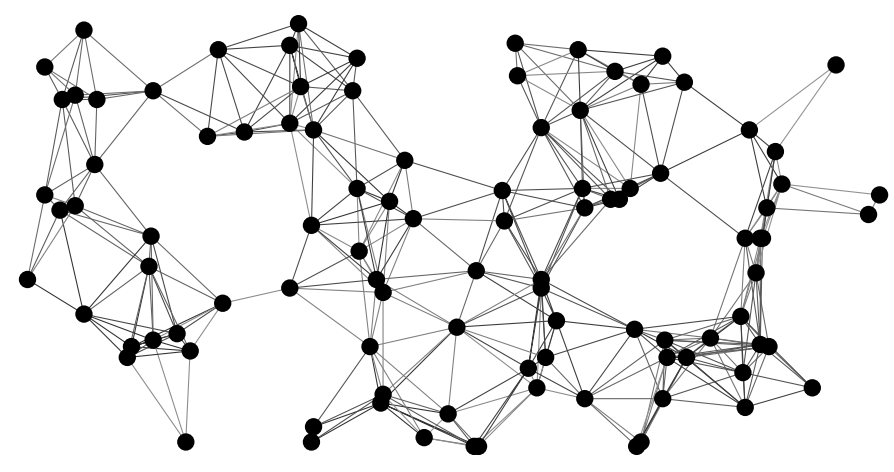

Fig. 2. A graph with average degree 8 .

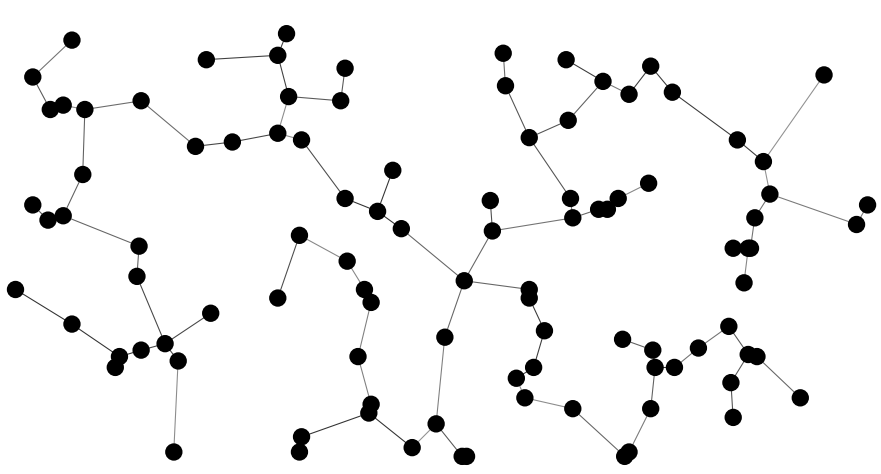

Fig. 3. Minimum spanning tree of graph in Fig. 2.

It also well-known that $M S T(G)$ is always strongly connected for a strongly connected graph $G$. Hence, in [9] the authors define the range adjustment as follows:

$$
\forall u \in V \quad r(u)=\max \left\{d(u, v) \mid v \in V \wedge(u, v) \in E_{m s t}\right\} .
$$

That means that each node chooses to reduce its range by just covering its neighbors in MST. We denote by $M S T^{*}(G)=G_{r}$ the graph with modified ranges by using MST edges. It is clear that $M S T(G)$ is included in $M S T^{*}(G)$ $\left(E_{m s t} \subseteq E_{r}\right.$ ) and then $M S T^{*}(G)$ is strongly connected. This protocol is called MTCP (MST Topology Control Protocol) in the remaining of this paper. It applies Prim's algorithm to construct a minimum spanning tree.

Wieselthier et al. have proposed in [9] two other globalized greedy heuristics for the minimum-energy broadcast problem. They are called BLU and BIP belong to the family of broadcast oriented protocols.

The BLU heuristic (Broadcast Least-Unicast-cost) applies the Dijkstra's algorithm. It merges low-energy unicasts from the source node to all other nodes in a single tree that is used instead of MST. In this case, power efficient routing protocols [22], [21] can be used to generate the basic structure. The BIP (Broadcast Incremental Power) is a modified version of the Prim's algorithm's where we consider additional cost in order to cover new nodes. The next node $v$ in BIP is selected to minimize the additional power (either by increasing 


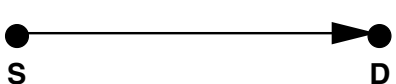

(a)

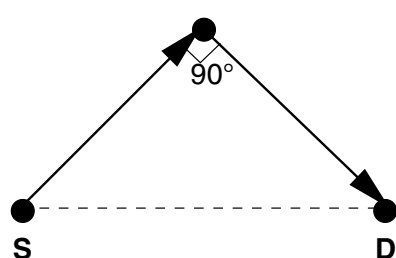

(b)

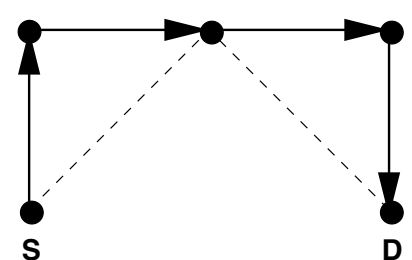

(c)

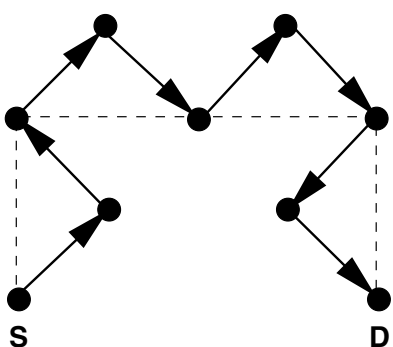

(d)

Fig. 1. Configuration with same energy consumption for $\alpha=2$ and $c=0$.

transmission power at one already transmitting node or by changing $r(u)=0$ to $r(u)=d(u, v)$ at one of MST neighbors). Although the authors [9] use an energy model with constant $c=0$, BIP fits well with the general model with arbitrary constant.

The authors [9] proposed also the "sweep" operation for removing some unnecessary transmissions, which is illustrated Fig. 4. A node $u$ whose communication area is covered by one of its neighbors (i.e. $\exists v \in N(u)$ such that $d(u, v)+r(u) \leq$ $r(v)$ ) may choose a null range.

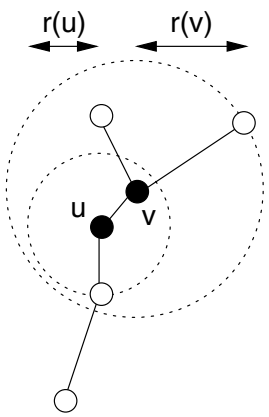

Fig. 4. Communication area of node $u$ is covered by node $v$.

There are some improvements of BIP algorithm but always in globalized manner and with an energy model using constant $c=0$ [2], [18], [19]. Wan et al. [19] gave analytical performance of BIP and showed that the approximation ratio of MST is bounded by 12. Liang [11] showed that BIP algorithm can have $\Omega(n)$ performance ratio with respect to the optimum algorithm in the worst case. They propose a sophisticated globalized solution with better performance ratio, but did not evaluate its average case performance. Mark et al. [18] proposed a generic search based globalized protocol for constructing the minimum power tree and claimed about $10 \%$ improvement over BIP.

Other works lead to approximation algorithm for the problem of minimizing the total power with a constant performance guarantee. For instance Lloyd et al. [8] propose a globalized algorithm which builds a 2-node-connected graph and assume an arbitrary energy model.

Lindsey and Raghavendra [17] proposed an algorithm which is not based on tree construction but still achieves the broadcast with less than $25 \%$ more energy consumption than the optimal solution. Their broadcasting protocol is the following. The source node simply sends a message to a central node (that is closest to all other nodes) by using power efficient routing protocol and the central node transmits the message to all other nodes with a single message. It is obvious that this protocol is not localized for designation of the central node. Moreover, this scheme has good results only for an energy consumption using $\alpha=2$ (the authors use an energy model with $c=0$ ) and is not efficient for higher exponents.

In our localized approach, we use the relative neighborhood graph (RNG) [23]. RNG was already applied for solving problems in wireless networks. For instance, [24] applied it to minimize the number of messages needed for broadcasting in one-to-one unit graph model. Borbash and Jennings [25] described the localized construction of RNG in details and proposed to use it as connected topology to minimize node degrees, hop-diameter, maximum transmission radius and the number of biconnected components. However, [25] do not describe the use of RNG in solving any specific problem.

\section{LOCALIZED PROTOCOLS}

\section{A. RNG Topology Control Protocol (RTCP)}

The main disadvantage of existing protocols is that algorithms are not localized. Our proposal is to substitute MST by the relative neighborhood graph (RNG) [23]. Let $V$ be a set of vertices and $G=(V, E)$ the induced graph with maximal range. The relative neighborhood graph of $G$ is denoted by $R N G(G)=\left(V, E_{r n g}\right)$ and if defined by:

$$
\begin{array}{r}
E_{r n g}=\{(u, v) \in G \mid \nexists w \in V \quad(u, w),(w, v) \in G \\
\wedge d(u, w) \leq d(u, v) \wedge d(v, w) \leq d(u, v)\} .
\end{array}
$$

This condition is illustrated Fig. 5: an edge $(u, v)$ belongs to the RNG if there does not exists a node $w$ is gray area.The gray area is the intersection of two circles centered at $u$ and $v$ and with radius $d(u, v)$. We can see in Fig. 6 the RNG of graph given Fig. 2. In this example, and typically in general, the average degree of RNG is around 2.5 (against 2 for MST).

Analogously, the range adjustment can be defined in order that each node can reach all its neighbors in $R N G(G)$ :

$$
\forall u \in V \quad r(u)=\max \left\{d(u, v) \mid v \in V \wedge(u, v) \in E_{r n g}\right\} .
$$




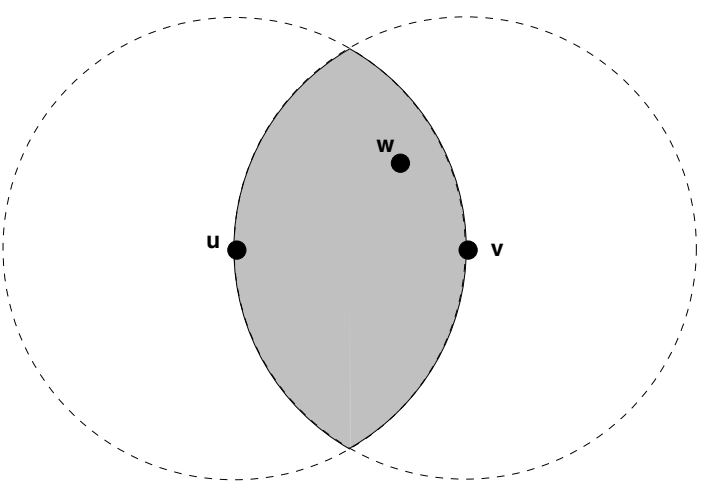

Fig. 5. The edge $(u, v)$ is not in RNG because of $w$.

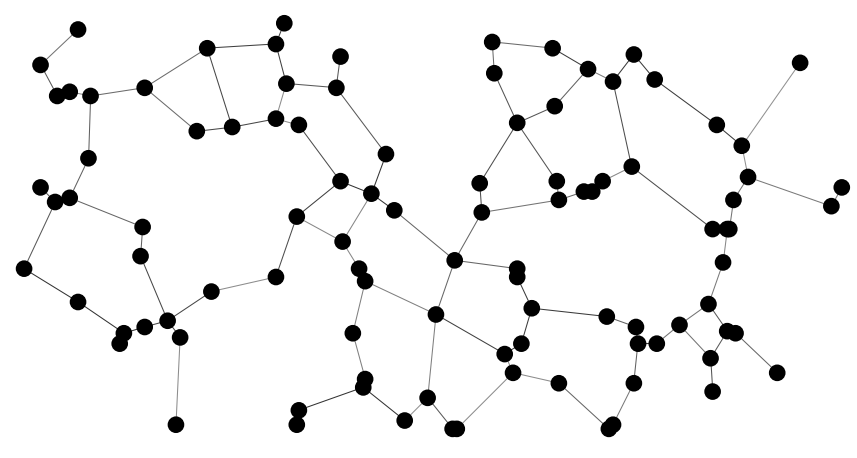

Fig. 6. Relative neighborhood graph for graph in Fig. 2.

The induced graph $G_{r}$ is denoted by $R N G^{*}(G)$. It is wellknown that $M S T(G)$ is included in $R N G(G)$ [1] and it is easy to see that $R N G(G)$ is a subset of $R N G^{*}(G)$. For a strongly connected graph $G$, the connectivity of $R N G^{*}(G)$ is then guaranteed. We will refer to this protocol as RTCP (RNG Topology Control Protocol).

The RNG can be deduced locally by each node by using only the distance with its neighbors. With positing system (like GPS), nodes need to send periodically an "HELLO" message with coordinates. In this way, each node maintains a neighborhood list with neighbor locations that allows to determine whether or not an edge is in RNG. In this case, we need only 1-hop information.

We can observe that if nodes do not have positioning system, nodes can achieve RNG edges determination if they are able to determine mutual distances (for instance by using signal strength or time delay information). Every node sends in its HELLO message the list of its neighbors with distances. Hence, RNG construction does not require more information or different HELLO message as required to construct MST. More information about RNG construction can be found in [25], [24]. The information required to make decision is 2-hop distance information. In both cases, with GPS or with distance ability, the algorithm for RNG edges determination is localized (with a knowledge of 1 or 2 hops distance neighborhood).

The connectivity of RNG assures that all nodes receive the message for any choice of the source node. We now discuss

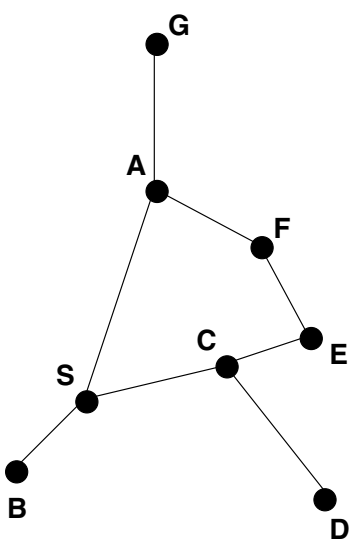

Fig. 7. Example of RNG graph for broadcast.

the adaptation and some improvements of RTCP to derive a broadcast oriented protocol.

\section{B. RNG Broadcast Oriented Protocol (RBOP)}

A topology control protocol aims to reduce transmission range while maintaining connectivity. For broadcasting, this kind of protocol provides an efficient energy sasving even by using blind flooding, but we can enhance the energy savings further. The idea is that when receiving a message from given neighbor it is not needed to reach this node, or nodes already covered by this node, by our retransmission.

Let us consider the graph illustrated Fig. 7 where nonRNG edges have been omitted. If the node $S$ wants to send a broadcast message, it transmits it with the minimal range which allows to join its RNG-neighbors (namely $A, B$ and $C)$. Then $S$ emits its message with the range $d(S, A)$ and $A$, $B$ and $C$ receive the message. Hence $S$ forwards the message with the range $d(A, S)$ (since $A$ is its further RNG-neighbor). It is quite obvious that $A$ could adjust its range to $d(A, G)$ since $S$ already has the message. In similar way, $B$ does not have to retransmit the message since all its RNG-neighbors $(S)$ have already received the message. This new "trick" is similar to neighbor elimination scheme [3], [26] but only applied to neighbors in RNG graph.

Let us continue the broadcast. The node $C$ also receives the message from $S$. According to preceding remark, $C$ resends the message with range $d(C, D)$. It is received by nodes $D, E$ but also $F$ even if it is not a RNG-neighbor. Hence $F$ receives the broadcast from a non-RNG edge. In this case, it is better that $F$ applies neighbor elimination but does not retransmit the message immediately. In fact, most of the time nodes get the message from one of its neighbors in RNG, hence by processing only neighbor elimination for transmissions coming from non-RNG edges the RNG-neighborhood will be smaller. In our example, $F$ eliminates $E$ for this broadcast message. The set of remaining neighbors for $F$ contains only $A$. At the same time $E$ decides not to send the message since all its RNG-neighbors are eliminated with message from $C$. It is the same case for $D$. When $A$ forwards the message, $F$ 


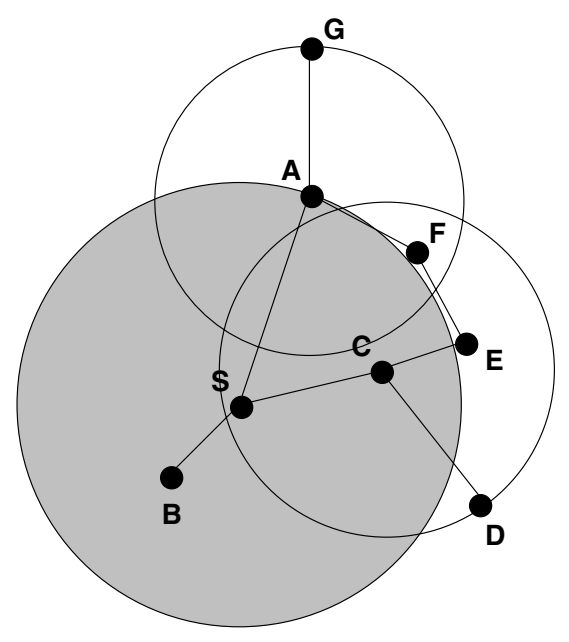

Fig. 8. Broadcast from $S$ with neighbor elimination.

and $G$ eliminate $A$ from their respective neighborhood list and terminate the protocol for this broadcast since their lists are empty. The broadcast is accomplished by 3 transmissions: from $S$ with radius $d(S, A)$, from $C$ with radius $d(C, D)$ and $A$ with radius $d(A, G)$ (see Fig. 8).

The localized improved protocol is then the following one:

1) the source node $u$ of a broadcast emits its message with determined range $r(u)$ from RTCP,

2) when receiving a new broadcast message:

a) if the emitter is a RNG-neighbor: the node calculates the furthest of its RNG-neighbors that did not receive this message. The node resends the message according to this range or ignores the message if all its RNG-neighbors have received the message,

b) otherwise, the node generates, for this broadcast, the list of RNG-neighbors that have not received this message. After a given timeout, if the neighbor list is not empty (neighbors can be removed by action $3 \mathrm{~b}$ ), the node retransmits the message with a range allowing to reach furthest neighbor in the associated list,

3) when receiving an already received message:

a) the node ignores the message if it has already forwarded it,

b) the node removes nodes that received this message from the associated neighborhood list,

c) the message is ignored if the associated list is empty,

d) otherwise, if the message arrives on a RNG-edge, send the message with range allowing to reach furthest neighbor in the list of non-eliminated RNG neighbors.

In next section, we give simulation results for presented protocol, which is referred as RBOP (RNG Broadcast Oriented Protocol), and other protocols described in this and previous section.

\section{PERformance eVAluation}

In our simulations, we compare four protocols. Two of these protocols are globalized: MST Topology Control Protocol (MTCP) and the Broadcast Incremental Power (BIP) from [9] (enhanced with the sweep operation). The two other protocols are the two new localized algorithms we propose: RNG Topology Control Protocol (RTCP) and RNG Broadcast Oriented Protocol (RBOP). In order to permit comparison with works in the literature, we use two different energy models: $\alpha=2, c=0$ and $\alpha=4, c=10^{8}$.

The parameters of our simulations are the following. The number of nodes $n$ is always 100 and nodes are static. The maximum communication radius $R$ is fixed to 250 meters. The MAC layer is assumed to be ideal. Nodes are randomly placed in a square area whose size is computed to obtain a given density (from 6 nodes per communication zone to 30). The timeout used in neighbor elimination scheme in RBOP is fixed to three times the duration of a message sending. Only connected sets are retained. For each measure, 5000 broadcasts have been run.

Because of ideal MAC layer and nature of protocols, we are sure that all nodes receive broadcasted messages. Hence, the "reachability" is always $100 \%$. The observed parameter is the energy consumption (according to both energy models). For each broadcast, we calculate the total energy consumption:

$$
E_{\text {total }}=\sum_{u \in V} E(u),
$$

where $E(u)$ depends of the transmission radius as explained in Section II. This total energy consumption $E$ is compared with total energy consumption needed for blind flooding protocol with maximal range:

$$
E_{\text {flooding }}=n \times\left(R^{\alpha}+c\right) .
$$

For the four considered protocols, we computed the average expended energy ratio (EER) that is defined by:

$$
E E R=\frac{E_{\text {total }}}{E_{\text {flooding }}} \times 100 .
$$

In Fig. 9 and Tables I and II, we show the comparison of saved energy for the four protocols and the two energy models. The average degree varies with density (in nodes per communication nodes) but is not exactly the same because of border effect.

We can observe that localized RBOP has quite close performance to the performance of globalized MTCP protocol. The difference does not exceed $25 \%$. This fact illustrates that localized algorithms can be very competitive with globalized one. But it is not surprising to see that the best algorithm is globalized: you can make better choice with full knowledge of the network than with a local view of the network. Hence, in both energy models, BIP spends $50 \%$ less energy than RBOP in average case. This overhead during broadcasting task for our 


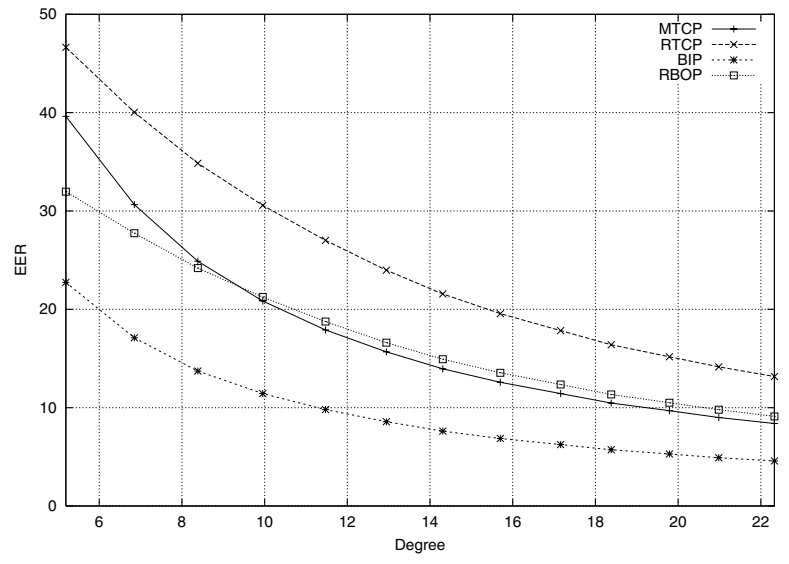

(a) $\alpha=2 \quad c=0$

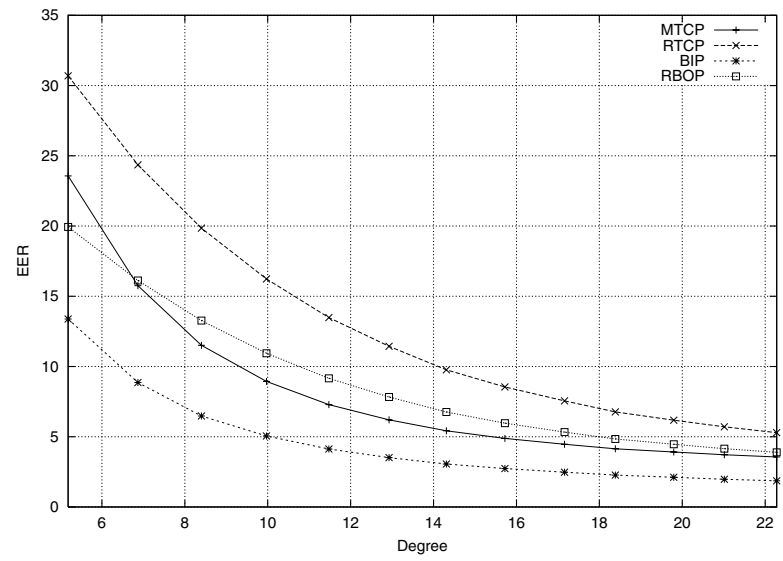

(b) $\alpha=4 \quad c=10^{8}$

Fig. 9. Expended energy ratio comparison.

\begin{tabular}{r|r||r|r|r|r}
\multicolumn{1}{c||}{} & \multicolumn{4}{c}{ EER } \\
\cline { 4 - 6 } density & degree & MTCP & RTCP & BIP & RBOP \\
\hline 6 & 5.194 & 39.621 & 46.646 & 22.746 & 31.967 \\
8 & 6.849 & 30.645 & 40.041 & 17.100 & 27.742 \\
10 & 8.386 & 24.883 & 34.853 & 13.722 & 24.189 \\
12 & 9.956 & 20.843 & 30.573 & 11.435 & 21.252 \\
14 & 11.473 & 17.898 & 27.014 & 9.804 & 18.761 \\
16 & 12.945 & 15.677 & 23.983 & 8.564 & 16.613 \\
18 & 14.308 & 13.944 & 21.559 & 7.618 & 14.927 \\
20 & 15.701 & 12.579 & 19.565 & 6.859 & 13.549 \\
22 & 17.159 & 11.432 & 17.838 & 6.241 & 12.354 \\
24 & 18.383 & 10.467 & 16.399 & 5.711 & 11.344 \\
26 & 19.789 & 9.695 & 15.174 & 5.288 & 10.502 \\
28 & 20.984 & 9.000 & 14.152 & 4.913 & 9.790 \\
30 & 22.329 & 8.394 & 13.163 & 4.577 & 9.108
\end{tabular}

TABLE I

EXPENDED ENERGY RATIO FOR $\alpha=2, c=0$.

\begin{tabular}{r|r||r|r|r|r}
\multicolumn{2}{c||}{} & \multicolumn{4}{c}{ EER } \\
\cline { 3 - 6 } density & degree & MTCP & RTCP & BIP & RBOP \\
\hline 6 & 5.185 & 23.572 & 30.689 & 13.383 & 19.939 \\
8 & 6.868 & 15.755 & 24.354 & 8.858 & 16.125 \\
10 & 8.401 & 11.506 & 19.851 & 6.479 & 13.269 \\
12 & 9.969 & 8.936 & 16.232 & 5.056 & 10.940 \\
14 & 11.476 & 7.284 & 13.485 & 4.126 & 9.159 \\
16 & 12.928 & 6.203 & 11.441 & 3.514 & 7.832 \\
18 & 14.309 & 5.426 & 9.767 & 3.060 & 6.761 \\
20 & 15.722 & 4.882 & 8.548 & 2.734 & 5.965 \\
22 & 17.160 & 4.468 & 7.552 & 2.480 & 5.330 \\
24 & 18.386 & 4.145 & 6.767 & 2.271 & 4.841 \\
26 & 19.795 & 3.916 & 6.181 & 2.116 & 4.464 \\
28 & 21.015 & 3.719 & 5.704 & 1.976 & 4.151 \\
30 & 22.278 & 3.563 & 5.287 & 1.862 & 3.894
\end{tabular}

TABLE II

EXPENDED ENERGY RATIO FOR $\alpha=4, c=10^{8}$. localized RBOP protocol compensates the network load which is needed to achieve full knowledge of network in globalized solutions. It can be also observed that expended energy ratios (EER) for both protocols decrease with increased density, or increased values for $\alpha$ and $c$. It can be observed that BIP protocol has roughly the same performance in both energy models. On the other hand, EER of RBOP decreased with increased $\alpha$ and $c$.

The difference between BIP and RBOP grows when density rises. This is due to the fact that in RNG graph the average degree is constant (around 2.5 neighbors). It means that the height of the spanning tree generated by the flooding with RBOP is constant (for sufficiently high density) and that the average transmission radius is inversely proportional to density. It follows that for high density network it could be more efficient to consider larger communication area than in RNG range adjustment. This give us a way for future improvements that are explained in the coming conclusion section.

\section{CONCLUSION}

In this paper we presented a localized RNG based minimum energy broadcast RBOP protocol that competes with globalized BIP protocol [9] or minimum spanning tree based protocol. This achievement can be explained by observing that the nature of broadcasting task differs from the nature of routing task. While MST structure closely resembles energy requirements of a routing task, it does not necessarily capture the structural properties in case of broadcasting. Increased transmission radius beyond the value of furthest uncovered neighbor in any MST like or RNG structure does not necessarily increase the overall energy consumption. It is quite possible that a small increase beyond longest RNG edge will reach several new neighboring nodes, and therefore the energy needed per one reached node may actually decrease (in one-to-all communication model). This explanation for good performance of RBOP protocol actually gives direction for further improvements in its performance, which is currently investigated by our group. The value $r(u)$ in RBOP is actually the minimum possible transmission radius which is required to maintain connectivity of the broadcast process. We can sort all neighbors (not already eliminated) by their distance 
to $u$, and consider ratios $E(u) / M(u)$, where $E(u)$ is the transmission power from the energy model, and $M(u)$ is the number of non-eliminated neighbors reached by transmitting with transmission radius equal to the distance $d$ (note that $E(u)$ and $M(u)$ depend on the distance of selected neighbor to $u$ ). The optimal ratio, constrained by distances $\geq r(u)$, will then be selected. We are also exploring other directions for further improvements.

Networks where nodes can only choose between active (range set to maximum) or inactive state (range set to zero) are special cases which have been addressed by several works. Dominating sets protocols [5], [26] can be seen as a solution for the min assignment problem for this case. MPR (Multipoint relaying) broadcast [4] and stochastic flooding [27] can be seen as energy-efficient broadcast protocols for active-inactive power assignment networks. Some ideas of these protocols, or some combinations between RBOP and these protocols may allow to improve our present results.

\section{REFERENCES}

[1] P. Bose, P. Morin, I. Stojmenović, and J. Urrutia, "Routing with guarantee delivery in ad hoc networks," ACM/Kluwer Wireless Networks, vol. 7, no. 6, pp. 609-616, 2001.

[2] T. Chu and I. Nikolaidis, "Energy efficient broadcast in mobile ad hoc networks," in Proc. Ad-Hoc Networks and Wireless (ADHOC-NOW), Toronto, Canada, 2002, pp. 177-190.

[3] W. Peng and X. Lu, "On the reduction of broadcast redundancy in mobile ad hoc networks," in Proc. Annual Workshop on Mobile and Ad Hoc Networking and Computing (MobiHoc'2000), Boston, Massachusetts, USA, 2000.

[4] A. Qayyum, L. Viennot, and A.Laouiti, "Multipoint relaying for flooding broadcast messages in mobile wireless networks," in Proc. 35th Annual Hawaii International Conference on System Sciences (HICSS'02), Hawaii, USA, 2002

[5] J. Wu and H. Li, "A dominating-set-based routing scheme in ad hoc wireless networks," in Proc. 3rd Int'l Workshop Discrete Algorithms and Methods for Mobile Computing and Comm (DIALM'99), Seattle, USA, 1999, pp. 7-14.

[6] L. Kirousis, E. Kranakis, D. Krizanc, and A. Pelc, "Power consumption in packet radio networks," in Proc. 14th Symp. on Theoretical Computer Science (STACS'97), ser. Lecture Notes in Computer Science, R. Reischuk and M. Morvan, Eds., vol. 1200. Hansestadt Lübeck, Germany: Springer-Verlag, Berlin, 1997, pp. 363-374.

[7] A. Clementi, P. Penna, and R. Silvestri, "The power range assignment problem in radio networks on the plane," in Proc. 17th Symp. on Theoretical Computer Science (STACS'O0), ser. Lecture Notes in Computer Science, H. Reichel and S. Tison, Eds., vol. 1770. Lille, France: Springer-Verlag, Berlin, 2002, pp. 651-660.

[8] E. Lloyd, R. Liu, M. Marathe, R. Ramanathan, and S. Ravi, "Algorithmic aspects of topology control problems for ad hoc networks," in Proc. Annual Workshop on Mobile and Ad Hoc Networking and Computing (MobiHoc'2002), Lausanne, Switzerland, 2002.

[9] J. Wieselthier, G. Nguyen, and A. Ephremides, "On the construction of energy-efficient broadcast and multicast trees in wireless networks," in Proc. IEEE Infocom'2000, Tel Aviv, Israel, 2000, pp. 585-594.
[10] O. Eğecioğlu and T. Gonzalez, "Minimum-energy broadcast in simple graphs with limited node power," in Proc. IASTED Int. Conf. on Parallel and Distributed Computing and Systems, Anaheim, Canada, 2001, pp. 334-338.

[11] W. Liang, "Constructing minimum-energy broadcast trees in wireless ad hoc networks," in Proc. Annual Workshop on Mobile and Ad Hoc Networking and Computing (MobiHoc'2002), Lausanne, Switzerland, 2002.

[12] J. Cartigny, D. Simplot, and I. Stojmenović, "Localized energy efficient broadcast for wireless networks with directional antennas," in Proc. Mediterranean Ad Hoc Networking Workshop (MED-HOC-NET'2002), Sardegna, Italy, 2002.

[13] A. Spyropoulos and C. Raghavendra, "Energy efficient communications in ad hoc networks using directional antennas," in Proc. IEEE Infocom'2002, New-York, USA, 2002.

[14] J. Wieselthier, G. Nguyen, and A. Ephremides, "Energy-limited wireless networking with directional antennas: the case of session-based multicasting," in Proc. IEEE Infocom'2002, New-York, USA, 2002.

[15] A. Benlarbi, J.-C. Cousin, R. Ringot, A. Mamouni, and Y. Leroy, "Interferometric positioning systems by microwaves," in Proc. Microwaves Symp. (MS'2000), Tetuan, Morocco, 2000.

[16] S. Banerjee and A. Misra, "Minimum energy paths for reliable communication in multi-hop wireless networks," in Proc. Annual Workshop on Mobile and Ad Hoc Networking and Computing (MobiHoc'2002), Lausane, Switzerland, 2002.

[17] S. Lindsey and C. Raghavendra, "Energy efficient broadcasting for situation awareness in ad hoc networks," in Proc. Int. Conf. Parallel Processing (ICPP'01), Valencia, Spain, 2001.

[18] R. M. II, A. Das, M. El-Sharkawi, P. Arabshahi, and A. Gray, "Minimum power broadcast trees for wireless networks: optimizing using the viability lemma," in Proc. IEEE Int. Symp. on Circuits and Systems, Scottsdale, USA, 2002, pp. 245-248.

[19] P.-J. Wan, G. Calinescu, X.-Y. Li, and O. Frieder, "Minimum energy broadcast routing in static ad-hoc wireless networks," ACM/Kluwer Wireless Networks, 2002, to appear.

[20] L. Feeney, "An energy-consumption model for performance analysis of routing protocols for mobile ad hoc networks," ACM J. of Mobile Networks and Applications, vol. 3, no. 6, pp. 239-249, 2001.

[21] V. Rodoplu and T. H. Meng, "Minimum energy mobile wireless networks," IEEE J. Selected Area in Comm., vol. 17, no. 8, pp. 1333-1344, 1999.

[22] X.-Y. Li and P.-J. Wan, "Constructing minimum energy mobile wireless networks," ACM Mobile Computing and Communication Reviews, vol. 5, no. 4, pp. 55-67, 2001.

[23] G. Toussaint, "The relative neighborhood graph of finite planar set," Pattern Recognition, vol. 12, no. 4, pp. 261-268, 1980.

[24] M. Seddigh, J. Gonzalez, and I. Stojmenović, "Rng and internal node based broadcasting algorithms for wireless one-to-one networks," $A C M$ Mobile Computing and Communications Review, vol. 5, no. 2, pp. 3744, 2001.

[25] S. Borbash and E. Jennings, "Distributed topology control algorithm for multihop wireless networks," in Proc. 2002 World Congress on Computational Intelligence (WCCI 2002), Honolulu, Hawaii, 2002.

[26] I. Stojmenović, M. Seddigh, and J. Zunic, "Dominating sets and neighbor elimination based broadcasting algorithms in wireless networks," IEEE Transactions on Parallel and Distributed Systems, vol. 13, no. 1, pp. 14-25, 2002.

[27] J. Cartigny and D. Simplot, "Border node retransmission based probalistic broadcast protocols in ad-hoc networks," in Proc. 36th Annual Hawaii International Conference on System Sciences (HICSS'03), Hawaii, USA, 2003. 